\title{
Mordedura por víbora de coral (Micrurus altirostris): primer caso en Uruguay
}

\author{
Carolina Juanena*, Pedro Saldun†, Banny Zelada ${ }^{\ddagger}$, Alba Negrin§, \\ Daniela Paciel", Santiago Carreira**
}

\section{Resumen}

Las mordeduras por ofidios del género Micrurus son infrecuentes a nivel mundial. En Uruguay los emponzoñamientos son exclusivamente por Bothrops alternatus y B. pubescens (crucera y yarará). Desde 1986 la notificación de casos de ofidismo es obligatoria ante el Centro de Información y Asesoramiento Toxicológico (CIAT), no habiéndose registrado casos por mordedura de Micrurus altirostris hasta este reporte clínico. Se presenta el primer caso clínico de mordedura por víbora de coral (Micrurus altirostris) en nuestro país, destacándose la gravedad del mismo dada la neurotoxicidad que presentó evolucionando a parálisis respiratoria que requirió ventilación mecánica invasiva. Buena evolución con el sostén de funciones vitales implementado, no requiriendo neostigmina, logrando extubación y reversión completa del cuadro al cuarto día. El antídoto de este emponzoñamiento (suero antielapídico) presenta limitada disponibilidad a nivel mundial, no contando con el mismo en nuestro país, por lo que en casos graves, particularmente con falla respiratoria, la asistencia ventilatoria mecánica es el principal pilar terapéutico.

Palabras clave: Mordeduras de serpientes

Serpientes de coral

Respiración artificial

Suero antielapídico

Key words: $\quad$ Snake bites

Coral snakes

Artificial respiration

Antielapidic serum.

\footnotetext{
* Médica Asistente Grado 2. Departamento de Toxicología, Hospital de Clínicas, Facultad de Medicina, Universidad de la República. † Médico Unidad de Medicina Intensiva. Hospital de Florida "Dr. Raúl Amorin Cal”. ASSE. Uruguay. Ex Asistente de Cátedra de Medicina Intensiva. Facultad de Medicina. Uruguay.

$\ddagger$ Posgrado de Toxicología. Departamento de Toxicología, Hospital de Clínicas, Facultad de Medicina, Universidad de la República, Uruguay. § Prof. Agda. Departamento de Toxicología, Hospital de Clínicas, Facultad de Medicina, Universidad de la República, Uruguay.

I Médica con función de alta dedicación. Prof. Adj. Cátedra de Enfermedades Infecciosas. Facultad de Medicina, Universidad de la República, Uruguay.

** Investigador Laboratorio de Sistemática e Historia Natural de Vertebrados, Instituto de Ecología y Ciencias Ambientales, Facultad de Ciencias, Universidad de la República, Uruguay. Sección Herpetología, Museo Nacional de Historia Natural, Uruguay.

Correspondencia: Dra. Carolina Juanena. Correo electrónico: dracarolinajuanena@hotmail.com
} 


\section{Introducción}

En América el promedio de accidentes ofídicos es de 6,2 por 100.000 habitantes, siendo causados mayoritariamente por animales del género Bothrops ${ }^{(1)}$. Las mordeduras por elápidos del género Micrurus son infrecuentes a nivel mundial, a modo de ejemplo representan menos del $1 \%$ en Brasil ${ }^{(2)}$. En Uruguay, donde los accidentes ofídicos son de tan solo 2,4 por 100.000, los emponzoñamientos son exclusivamente por Bothrops alternatus y $B$. pubescens (crucera y yarará) con una incidencia anual de aproximadamente $70 \operatorname{casos}^{(1,3,4)}$. Desde 1986, la notificación de casos de ofidismo es obligatoria ante el Centro de Información y Asesoramiento Toxicológico (CIAT), no habiéndose registrado casos por mordedura de Micrurus altirostris hasta este reporte clínico ${ }^{(5,6)}$.

La familia Elapidae, de distribución global, está representada en Uruguay únicamente por $M$. altirostris (popularmente víbora de coral). Se distribuye en todo el territorio nacional y se caracteriza por la presencia de anillos completos alrededor del cuerpo: anillos negros agrupados de a tres, separados por anillos amarillos (tríadas) y entre estos se encuentra un anillo rojo ${ }^{(7)}$ (figura 1). Su veneno es predominantemente neurotóxico pudiendo causar bloqueo neuromuscular, que en alrededor de $4 \%-5 \%$ de los casos pueden conllevar falla respiratoria $^{(2)}$. El antídoto de este emponzoñamiento (suero antielapídico) presenta limitada disponibilidad a nivel mundial, por lo que en casos graves, particularmente con falla respiratoria, la asistencia ventilatoria mecánica es el principal pilar terapéutico ${ }^{(8,9)}$. En este trabajo se presenta el primer caso clínico de emponzoñamiento por M. altirostris en nuestro país, destacándose la gravedad del mismo dada la neurotoxicidad que evolucionó a parálisis respiratoria, requiriendo ingreso a una unidad de medicina intensiva y ventilación mecánica invasiva.

\section{Caso clínico}

Hombre de 25 años, procedente de zona rural de Flori$\mathrm{da}$, sin antecedentes a destacar, mordido en el tercer dedo de mano izquierda al manipular durante horas a un ofidio que describe con anillos negros, rojos y amarillos. Comienza con dolor abdominal y náuseas, agregando aproximadamente a las cinco horas del accidente mareos, visión borrosa y diplopía, por lo que consulta en emergencia del Hospital de Florida. Al ingreso refiere mialgias, disfagia y dolor en sitio de mordedura. Al examen físico se observa una puntura no sangrante, con eritema y edema leve localizado en tercer dedo de mano izquierda sin equimosis (figura 2). Lúcido, con disartria, oculomotricidad sin alteraciones. Reflejo fotomotor y consensual conservados. Se consulta al CIAT, realizándose evaluación fotográfica del ofidio identificán-

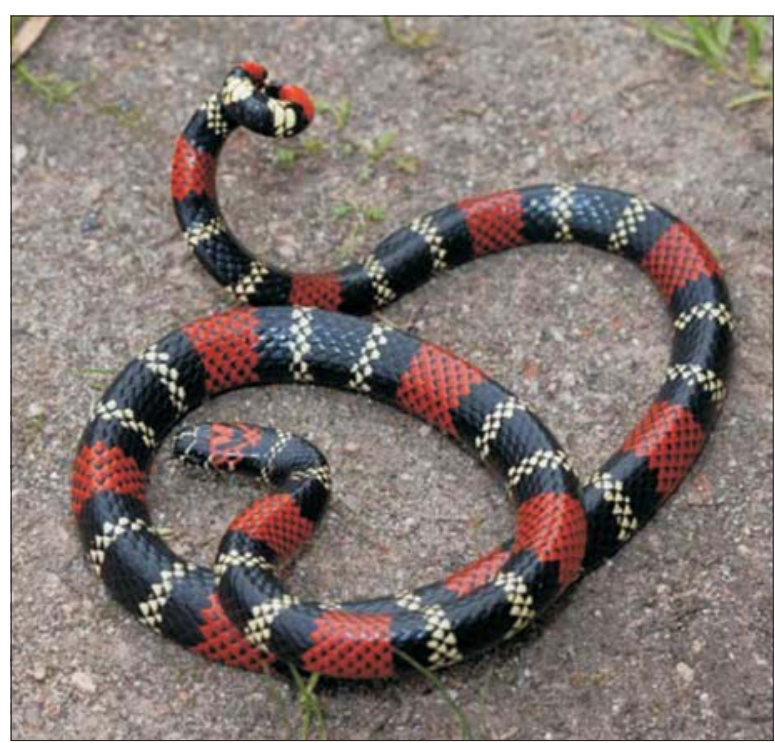

Figura 1. Ejemplar adulto de Mircurus altirostris. Presenta anillos completos alrededor del cuerpo, negros agrupados de a tres, separados por anillos amarillos (tríadas) y entre estos se encuentra un anillo rojo.

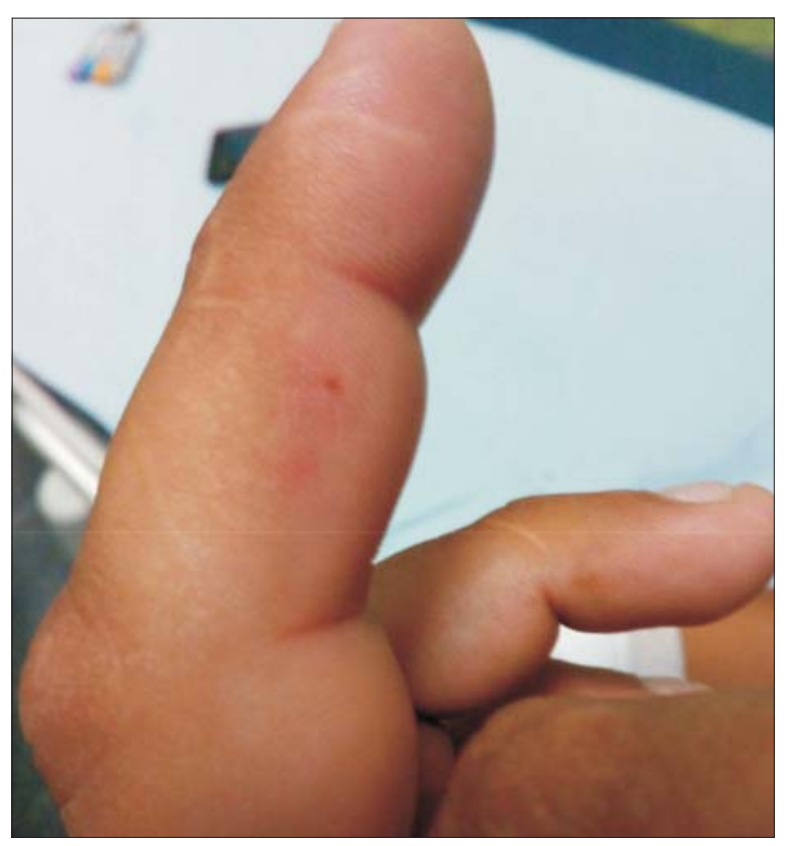

Figura 2. Puntura no sangrante, eritema y edema leve localizado en tercer dedo de mano izquierda sin equimosis.

dose el ejemplar como víbora de coral (M. altirostris) (figura 3). Ingresa a la Unidad de Tratamiento Intensivo (UTI) dada la progresión de síntomas, agregando ptosis palpebral bilateral y parálisis flácida de miembros (aproximadamente a las siete horas de evolución). Peoría de la dinámica respiratoria con tos inefectiva, por lo 
que se procede a intubación orotraqueal y asistencia ventilatoria mecánica. $\mathrm{Al}$ momento de decúbito a $0^{\circ}$ sufre obstrucción muscular de la vía aérea alta que revierte con la subluxación de la mandíbula. Laringoscopía directa sin edema de glotis ni evidencia de macroaspiración. Se inicia sedación continua en base a benzodiacepinas y opiáceos.

De la paraclínica (tabla 1) se destaca plaquetopenia a las 24 horas que revierte en cinco días. Crasis sin alteraciones. Creatinfosfoquinasa total (CPK) normal. Radiografía de tórax sin alteraciones.

Se mantiene con hemodinamia estable, buen intercambio gaseoso, sin sangrados, sin otras disfunciones. Buena adaptación a la ventilación mecánica. Se suspende sedación a las 24 horas del ingreso. En cuanto a lo neurológico, se mantiene vigil, persistiendo ptosis palpebral y cuadriparesia hasta el cuarto día de evolución, revirtiendo íntegramente. Mejoría franca de dinámica respiratoria, siendo extubado al cuarto día. Sin complicaciones posteriores, con egreso hospitalario al séptimo día.

\section{Discusión}

Este es el primer caso de mordedura por esta especie, descrito y documentado en nuestro país, que motivó consulta médica y tratamiento debido a su gravedad.

Las características del ofidio (cabeza pequeña, maxilar fijo, con colmillos de disposición anterior reducidos, 2-3 mm), junto a un comportamiento nunca agresivo, determinan la escasa probabilidad de una mordedura eficaz ${ }^{(5,7)}$. La manipulación prolongada del animal, tal como la referida en este caso, es la circunstancia favorecedora del accidente dado que tiene el comportamiento de aferrarse y "masticar" luego de la mordedura inicial, aumentando el tiempo de inoculación y la eficiencia de la mordedura ${ }^{(10)}$. En este caso la misma se produjo en un dedo de mano, siendo esta localización la citada más frecuentemente $^{(2,10)}$. Las manifestaciones locales suelen ser muy leves, observándose una o dos punturas, o incluso ninguna, refiriéndose dolor local de intensidad variable, con progresión proximal ${ }^{(2,9,11)}$. Los elementos neurológicos locales como parestesias se presentan en menos de la mitad de los casos ${ }^{(2,9,11,12)}$. En este caso se evidenció una única puntura, dolor y edema leve sin equi-

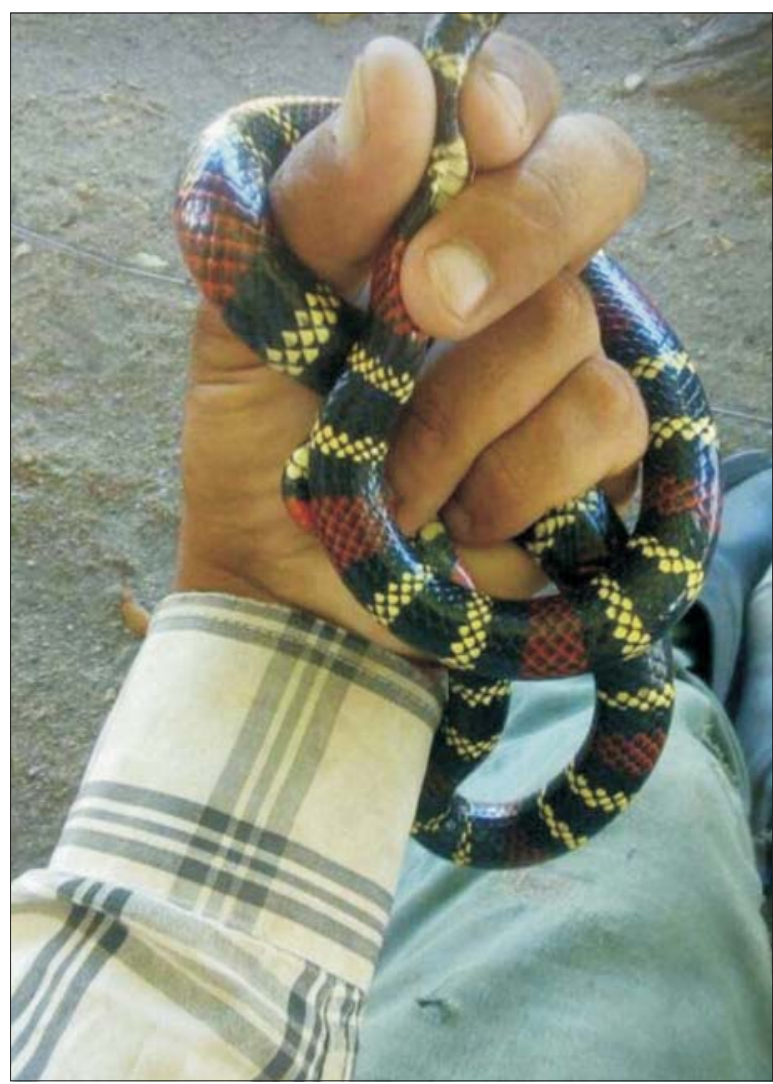

Figura 3. Foto recibida al momento de la consulta. Se observa la manipulación del ejemplar identificado como víbora de coral (Micrurus altirostris).

mosis, ni hemorragias o manifestaciones neurológicas locales. Probablemente la escasa relevancia de los signos locales en el sitio de la mordedura y la ausencia de síntomas iniciales determinó el retraso en la consulta médica. A nivel sistémico se describe la presencia de dolor abdominal, náuseas, sialorrea, diaforesis, mareos, somnolencia y euforia. Las manifestaciones neurológicas pueden iniciarse a los pocos minutos o retrasarse hasta las doce horas ${ }^{(2,10)}$. En este caso el paciente comenzó con síntomas neurológicos sistémicos a las cinco horas de la mordedura. La neurotoxicidad comienza típicamente con elementos de parálisis bulbar con alteración de la oculomotricidad, ptosis palpebral, disfagia, disar-

Tabla 1. Evolución de la paraclínica.

\begin{tabular}{lccccc}
\hline & 1er día & 2 di día & 3er día & $4^{\text {to }}$ día & $5^{\text {to día }}$ \\
\hline Plaquetas $\left(\mathrm{mm}^{3}\right)$ & 90.000 & 72.000 & 99.000 & 122.000 & 170.000 \\
CK (U/L) & 67 & 62 & - & - & - \\
Tasa protrombina (\%) & 76 & 83 & 75 & 68 & - \\
\hline
\end{tabular}


tria y puede progresar hacia la parálisis flácida descendente, parálisis muscular respiratoria, paro respiratorio y muerte ${ }^{(10,13,14)}$. Eso se debe a que el veneno del género produce neurotoxicidad por alteración de la placa motora, con un mecanismo de acción a nivel postsináptico (por inhibición competitiva de los receptores de Ach por alfa neurotoxinas) y a nivel presináptico (por inhibición en la liberación de Ach de las terminales presinápticas por la beta neurotoxina). Experimentalmente se describe que la neurotoxicidad del veneno de $M$. altirostris actúa a nivel postsináptico por alfa neurotoxina, lo que produce un síndrome "miasteniforme" agudo ${ }^{(15)}$. Otras complicaciones reportadas incluyen rabdomiólisis, un efecto trombino-símil que puede ser causa de coagulopatía y trombocitopenia ${ }^{(2)}$. En este caso se constató trombocitopenia leve que revirtió al quinto día, como se ha reportado en otros casos ${ }^{(16)}$.

En cuanto al tratamiento con antiveneno específico en revisiones de casos en Brasil, refieren que se debe indicar lo más precozmente posible en todo paciente con signos neurológicos iniciales ${ }^{(2)}$. Sin embargo, dicha indicación es controversial puesto que existen reportes de pacientes asintomáticos a quienes se administró el antiveneno y que desarrollaron tardíamente complicaciones neurológicas severas ${ }^{(11)}$. Por otra parte, hay reportes que describen la reversión tardía de los síntomas a pesar de la administración del antiveneno ${ }^{(2,10)}$. En nuestro caso, la demora desde la mordedura hasta la consulta, asociada a la rápida instalación posterior de las manifestaciones neurológicas severas (parálisis respiratoria), justificó el tratamiento de sostén realizado, alejando la oportunidad de indicación del tratamiento con antiveneno específico, a lo que se sumó la falta de disponibilidad del mismo en el medio. Destacamos, por otra parte, que en la actualidad en nuestra región el antiveneno disponible, procedente de Brasil, neutraliza pobremente el veneno de $M$. altirostris, según estudios experimentales ${ }^{(15,17)}$.

Otro de los recursos terapéuticos descritos en la literatura es el uso de neostigmina, principalmente para evitar la progresión del compromiso respiratorio por parálisis muscular y la necesidad de ventilación mecánica ${ }^{(18)}$. Cabe destacar que con el paciente ya en asistencia respiratoria mecánica y bajo sedación no es posible valorar adecuadamente la respuesta a este tratamiento, y que hay reportes en los que no se evidenció reversión de los síntomas neurológicos sumado a los eventuales efectos adversos graves que puede determinar su uso ${ }^{(10)}$. Estos factores hicieron que se desestimase su utilización el uso en este caso.

Finalmente, la intubación traqueal y la ventilación mecánica parecen mejorar los resultados en los pacientes con envenenamiento grave con falla respiratoria, siendo su indicación similar a otras enfermedades neu- romusculares agudas con potencial compromiso respiratorio, donde lo fundamental es el monitoreo clínico y de ser posible de las presiones inspiratorias y espiratorias $^{(2)}$. Una vez en asistencia respiratoria mecánica se evaluaron periódicamente las fuerzas del paciente para valorar la dinámica respiratoria en vistas a la desvinculación oportuna. En este paciente la misma fue relativamente precoz, al cuarto día, como se describe en reportes publicados previamente.

\section{Conclusiones}

Los casos por emponzoñamiento por el género Micrurus tienen una frecuencia baja a nivel mundial y regional, sin registros en Uruguay desde que el reporte es obligatorio.

En caso de envenenamiento se debe monitorizar la aparición de neurotoxicidad por al menos 24 horas, y de presentarla, se debe ingresar a unidad de medicina intensiva con posibilidad de intubación orotraqueal y ventilación mecánica invasiva.

Se debe considerar en los casos sin compromiso respiratorio el uso de anticolinesterásicos. Si bien los resultados clínicos del antiveneno son controversiales, su uso para evitar la progresión de la neurotoxicidad es recomendado por los centros toxicológicos de referencia.

\section{Abstract}

Micrurus snakebites are rather unusual globally. In Uruguay, poisoning cases are exclusively caused by Bothrops alternatus and B. pubescens ("crucera" and "yarará" crossed pit viper). Reporting poisoning to the Poison Information and Advice Service is mandatory since 1986, there being no cases reported of Micrurus altirostris bites until this clinical report. This study presents the first clinical case of coral snake bite (Micrurus altirostris) in our country, emphasizing its severity given the neurotoxicity that evolved into respiratory paralysis requiring invasive mechanical ventilation. Life support implemented resulted in a good evolution, no neostigmine being required, extubation and complete reversal of the clinical symptoms being achieved after four days. Availability of the poisoning antidote (antielapidic serum) is limited around the world, and it cannot be found in our country. Thus, in severe cases, in particular with respiratory failure, mechanical ventilation is the main therapeutic pillar.

\section{Resumo}

As picadas por ofídios do género Micrurus são pouco frequentes em todo o mundo. No Uruguai os envenenamentos são exclusivamente por Bothrops alternatus e $B$. pubescens ("urutu ou cruzeiro" e "jararaca pintada"). Desde 1986 a notificação de casos de ofidismo ao Centro de Información y Asesoramiento Toxicológico (CIAT) é 
obrigatória, não havendo registros por picada de Micrurus altirostris até o momento da descrição deste caso clínico. Apresenta-se o primeiro caso clínico de picada por cobra coral (Micrurus altirostris) no país, destacando-se a gravidade do mesmo devida à neurotoxicidade que apresentou e que evoluiu a paralisia respiratória sendo necessária realizar ventilação mecânica invasiva. A evolução foi boa com as medidas de suporte vital implementadas, não sendo necessária a utilização de neostigmina, com extubação e reversão completa do quadro no quarto dia. A disponibilidade do antídoto para este tipo de envenenamento (soro antielapídico) é limitada em todo o mundo não estando disponível no país por isso, especialmente nos casos graves com insuficiência respiratória, a ventilação mecânica é o principal pilar terapêutico.

\section{Bibliografía}

1. Chippaux J. Incidence and mortality due to snakebite in the Americas. PLoS Negl Trop Dis 2017; 11(6):e0005662. Disponible en: https://journals.plos.org/plosntds/article?id=10.1371/journal.pntd.0005662. [Consulta: 12 marzo 2018].

2. Bucaretchi F, Capitani E, Vieira R, Rodrigues C, Zannin M, Da Silva NJr, et al. Coral snake bites (Micrurus spp.) in Brazil: a review of literature reports. Clin Toxicol (Phila) 2016; 54(3):222-34.

3. Uruguay. Ministerio de Salud. Ofidismo. Montevideo: MSP, 2018. Disponible en: http://www.msp.gub.uy/publicaci\%C3\%B3n/ofidismo. [Consulta: 1 abril 2018].

4. Negrin A, Rosenberg N, Tortorella MN. Mordeduras de ofidios en intoxicaciones: casuísticas del Centro de Información y Asesoramiento Toxicológico. Montevideo: UCUR, 2011:59-64.

5. Carreira S, Negrin A, Tortorella MN, Pino A, Menéndez C. Ofidismo en Uruguay: especies peligrosas y características del accidente ofídico. Montevideo: CID/CEUR, 2006.

6. Purstcher H, Burger M, Savio E, Rodríguez Juanico L, Vila B, et al. Ofidismo y aracnidismo en el Uruguay: diagnóstico, complicaciones y tratamiento. Rev Méd Urug 1983; 7(1):1-28.

7. Carreira S, Meneghel M, Achaval F. Reptiles de Uruguay. Montevideo: DI.R.A.C, 2005.

8. Pizon A, Rhua A. Antidotes in depth. Antivenom: snakes Native (US) Venoms (Crotaline and Elapid). En: Hoffman
RS, Howland MA, Lewin NA, Nelson LS, Goldfrank LR. Goldfrank's toxicologic emergencies. 10 ed. New York: McGraw-Hill, 2015:1547-51.

9. Rhua A, Pizon A. Native (US) venomous snakes and lizards. En: Hoffman RS, Howland MA, Lewin NA, Nelson LS, Goldfrank LR. Goldfrank's Toxicologic Emergencies. 10 ed. New York: McGraw-Hill, 2015:1537-46.

10. Pardal P, Pardal J, Gadelha M, Rodrigues L, Feitosa D, Prudente A, et al. Envenomation by Micrurus coral snakes in the brazilian amazon region: report of two case. Rev Inst Med Trop Sao Paulo 2010; 52(6):333-7.

11. Wood A, Schauben J, Thundiyil J, Kunisaki T, Sollee D, Lewis-Younger C, et al. Review of Eastern coral snake (Micrurus fulvius fulvius) exposures managed by the Florida Poison Information Center Network: 1998-2010. Clin Toxicol (Phila) 2013; 51(8):783-8.

12. de Roodt A, De Titto E, Dolab J, Chippaux J. Envenoming by coral snakes (Micrurus) in Argentina, during the period between 1979-2003. Rev Inst Med Trop Sao Paulo 2013; 55(1):13-8.

13. Sasaki J, Khalil P, Chegondi M, Raszynski A, Meyer K, Totapally B. Coral snake bites and envenomation in children: a case series. Pediatr Emerg Care 2014; 30(4):262-5.

14. Gold B, Dart R, Barish R. Bites of venomous snakes. N Engl J Med 2002; 347(5):347-56.

15. de Abreu V, Leite G, Oliveira C, Hyslop S, Furtado M, Simioni L. Neurotoxicity of Micrurus altirostris (Uruguayan coral snake) venom and its neutralization by commercial coral snake antivenom and specific antiserum raised in rabbits. Clin Toxicol (Phila) 2008; 46(6):519-27.

16. Manock S, Suarez G, Graham D, Avila-Aguero M, Warrell D. Neurotoxic envenoming by South American coral snake (Micrurus lemniscatus helleri): case report from eastern Ecuador and review. Trans R Soc Trop Med Hyg 2008; 102(11):1127-32.

17. Tanaka G, Sant'Anna O, Marcelino J, Lustoza da Luz A, Teixeira da Rocha M, Tambourgi D. Micrurus snake species: Venom immunogenicity, antiserum cross-reactivity and neutralization potential. Toxicon 2016; 117:59-68.

18. Vital Brazil O, Vieira R. Neostigmine in the treatment of snake accidents caused by Micrurus frontalis: report of two cases (1). Rev Inst Med Trop Sao Paulo 1996; 38(1):61-7. 